\title{
Piperaquine- and Aspirin- Mediated Protective Role of HSP70 and HSP90 as Modes to Strengthen the Natural Immunity against Potent SARS-CoV-2
}

\author{
Fayez Saud Alreshidi 1,* ${ }^{10}$, Ibrahim Abdelmageed Ginawi ${ }^{2}{ }^{\mathbb{D}}$, Malik Asif Hussain ${ }^{3} \mathbb{1 D}^{\mathbb{D}}$, Jamal Mohammad \\ Arif ${ }^{4, *}$ (ID) \\ 1 Department of Family and Community Medicine, College of Medicine, University of Hail, Hail, 2440, Kingdom of Saudi \\ Arabia; fs.alreshidi@uoh.edu.sa (F.S.A.); \\ 2 Public Health Directorate, Hail, 2440, Kingdom of Saudi Arabia; iginawi71 @ gmail.com (I.A.G.); \\ 3 Department of Pathology, College of Medicine, University of Hail, 2440, Kingdom of Saudi Arabia; \\ mh.hussain@uoh.edu.sa (M.A.H.); \\ 4 Department of Biochemistry, College of Medicine, Shaqra University, Shaqra, 11961, Kingdom of Saudi Arabia; \\ jmarif@su.edu.sa (J.M.A.); \\ * Correspondence: jmarif@su.edu.sa (J.M.A.); fs.alreshidi@uoh.edu.sa (F.S.A.);
}

Scopus Author ID 6603906517

Received: 4.12.2020; Revised: 30.12.2020; Accepted: 2.01.2021; Published: 4.01.2021

\begin{abstract}
Since the emergence of COVID-19, the clinical manifestations are continually challenging the clinicians and scientists for the rapid development of a preventive solution of SARS-CoV-2 infections. Our hypothesis was inspired by the fact that the bats during powered flights generate heat shock responses via HSP70 and HSP90, which safeguard them from deadly viruses, including SARSCoV-2 harbored within the bats. Therefore, we hypothesized that HSP70 and HSP90 bind with S-ACE2 and/or AngII, thereby making ACE2 less available or unavailable for SARS-CoV-2, which may enhance immunity inhibiting the cytokine storm and acute respiratory distress syndrome (ARDS). Using molecular docking approaches, molecular dynamics (MD) simulation and CYP450 metabolism studies, detailed protein-protein (S-ACE2), complex protein-protein (HSP70/90-S-ACE2), and complex protein-ligands interactions (HSP70/90-S-ACE2-ASP/PIP/HYD) were carried out to establish the most probable protective mechanism of HSPs in SARS-CoV-2 mediated ARDS. Our results showed that out of many ligands tested, piperaquine (PIP) and Aspirin (ASP) interacted quite efficiently with HSP70/90-AngII compared to the HSP70/90-S-ACE2 with the formation of a relatively stable complex having excellent DockThor scores, $\Delta \mathrm{G}$, and $\mathrm{Kd}$ values. Further, it could be predicted that ASP-inducible HSP70/90 and PIP might work through binding with the accumulated AngII due to malfunctioning of ACE2, thereby preventing the cytokine storm ARDS with fewer chances of clot formation too in COVID-19 patients. However, these purely in silico findings need to be validated in the in vitro/in vivo experimentations for further insight into the proposed preventive mechanism(s).
\end{abstract}

Keywords: SARS-CoV-2; COVID-19; HSP70; HSP90; ACE2; S-protein.

(C) 2020 by the authors. This article is an open-access article distributed under the terms and conditions of the Creative Commons Attribution (CC BY) license (https://creativecommons.org/licenses/by/4.0/).

\section{Introduction}

Since the emergence of severe acute respiratory syndrome coronavirus 2 (SARS-CoV2) in December 2019, also known as 2019-nCoV, it has infected over 66 million people worldwide with $>1.5$ million deaths in the past one year as reported by the worldometers (www.worldometers.info). The exponential growth of COVID-19 cases, health infrastructure, availability, and preparedness of medical and paramedical staff to handle and contain the rapid 
spread of SARS-CoV-2 put many developed and developing countries under unexpected and challenging pressure. The age- and gender-based severity of COVID-19 infection, clinical symptoms, inter-individual response to the infection, mortality, and treatment outcomes had seen unexplained geographical variations among the countries [1]. Many clinical symptoms from COVID-19 infection have been observed, which includes but not limited to, e.g., cold, dry cough, mild to high fever, diarrhea, loss of taste and smell, severe respiratory illness, acute respiratory distress syndrome (ARDS), pulmonary embolism, pneumonia, blood clot formation, heart and brain strokes [2].

SARS-CoV-2 is a cytoplasmic replicating pathogen with positive-polarity, singlestranded RNA (ssRNA) genome consists of four major membrane-bound proteins viz., spike $(\mathrm{S})$, envelope $(\mathrm{E})$, and membrane $(\mathrm{M})$, and nucleocapsid $(\mathrm{N})$ glycoproteins along with few subsidiary proteins $[3,4]$. The S-protein consists of two domains, S1 and S2. S1 contains the N-terminal domain (NTD) and C-terminal domain (CTD), collectively known as receptor binding sites. S1-CTD is considered the receptor-binding domain (RBD) in SARS-CoV-2 and MERS-CoV [3]. As a vital part of the pathogen-host entry, these glycoproteins have gained high momentum towards designing immunotherapeutics and prophylactic vaccines against COVID-19 [5,6].

The most plausible mechanism has been depicted by the sequence analysis and several docking studies that S-protein binds tightly with angiotensin-converting enzyme 2 (ACE2) and thereby moving inside the host cell [7,8]. However, identifying the exact receptor binding site of SARS-CoV-2 with its host receptor ACE2 is underway [9-11].

Further, in a normal cell, ACE2 converts angiotensin II (AngII) into angiotensin I-VII, thereby preventing adverse effects on the cardiovascular system. However, in COVID-19 patients, due to S-ACE2 complex formation, it may not have enough cellular level of ACE2 to convert AngII to AngI-V, and as a result, serum AngII concentration increases. The increased concentration of AngII induces IL-6 family cytokines, followed by activation of the IL-6 amplifier that subsequently induces other proinflammatory cytokines and chemokines, leading to the onset of a cytokine storm. The cytokine storm causes acute respiratory distress syndrome (ARDS), responsible for the death of COVID-19 patients.

The scrupulous reports on bats' role as a reservoir for viruses causing various outbreaks, including Ebola, SARS, MERS, Nipah, Zika, Hendra, and 2019-nCoV viruses, have been published that reveals their unusual survival [12,13]. These viruses often cause severe morbidity and mortality in mammals, while bats remain clinically asymptomatic upon infection. Throughout evolution, bats may have gained novel functions permitting them to coexist with these viruses, thus evading the onset of severe pathology [14]. The most probable explanation for bats' immunity from these viruses' deleterious effects is a spurt of heat shock proteins (HSPs) during bats' powered flight [15].

HSPs, produced during high temperature and stress responses, are ubiquitous, evolutionary conserved proteins with multiple intracellular functions. The higher basal expression level of HSP70 and HSP90 could be a bat-unique, essential factor to modulate cellular stress and death. Consequently, bat cells survive prolonged heat treatment compared to other mammalian cells suggesting HSP expression in bats could be a vital adaption to intrinsic metabolic stresses such as powered flight. Further, up-regulated HSP70 and HSP90 during viral infection in bat species may serve as a cushion from the deleterious effects of various deadly viruses it harbors $[15,16]$. However, these viruses, when infecting another host like humans, who generally have lower levels of HSPs in normal physiological conditions (at 
$37^{\circ} \mathrm{C}$ ), pose severe and sometimes fatal effects. Further, simple drugs like sodium salicylate and aspirin have been shown to induce the HSP70 and HSP90 even at lower temperatures (20$22{ }^{\circ} \mathrm{C}$ ) in fish cell line CHSE-214 [17]. Additionally, HSP70 also inhibits cytokine storm induced from viruses like SARS by modulating the NFkB pathway [18].

Another interesting side-effect of SARS-CoV-2 is lung injury due to pulmonary embolism, which induces the formation of blot clots in both symptomatic and asymptomatic patients. Subsequently, it causes death due to heart and brain strokes [19]. Aspirin, an age-old pain-killer, and blood-thinning medicine, have shown promising results in some studies with reduced mortality from blood clots [20]; however, the underlying mechanism(s) are yet to be explored.

In light of aspirin's dual-action, it is worth exploring its potential in inhibiting the SARS-CoV-2 transmission in humans and/or the severity of clinical manifestations. We investigated the most probable protective mechanism of HSP70 and HSP90 in SARS-CoV-2 mediated ARDS using molecular docking approaches in the proposed research. We hypothesize that aspirin-induced HSP70 and HSP90 bind with S-ACE2 and/or AngII, thereby making ACE2 less available or unavailable for SARS-CoV-2, which subsequently may inhibit the cytokine storm and ARDS. Further, docking simulation of selected drugs viz., chloroquine, cinchonidine, cinchonine, erysolin, hydroxychloroquine, piperaquine, quinidine, quinine, sarcophine, solanidine, solanine, and spergualin with these complexes were also carried out, followed by MD simulation and metabolism study. The complex-ligand findings were found promising in explaining the protective role of HSPs in SARS-CoV-2 mediated ARDS. This molecular docking-based study uncovers novel drugs, piperaquine, and aspirin, to prevent 2019-nCoV transmission and could be used as a prophylactic and therapeutic drug candidate against SARS-CoV-2.

\section{Materials and Methods}

\subsection{Proteins $3 D$ structure retrieval, optimization, and minimization.}

3D structure of S-protein complexed with ACE2 (PDB ID: 6LZG), HSP70 (PDB ID: 4IO8), and HSP90 (PDB ID: 1YET) were retrieved from research collaboratory for structural bioinformatics (RCSB) protein data bank (PDB). All proteins were prepared as inputs for molecular docking by removing undesired heteroatoms, ions, and molecules. The CHARMm force field was assigned to them, and carried out energy minimization steps to remove steric clashes using the steepest descent gradient algorithm with an RMS gradient energy of 0.001 $\mathrm{kcal} / \mathrm{mol}[21]$.

\subsection{Ligands $3 D$ structure preparation, optimization, and minimization.}

2D structures of all ligands in SDF format were retrieved from the PubChem database of NCBI (http://pubchem.ncbi.nlm.nih.gov). Conversion of SDF to PDB format was achieved using BIOVIA discovery studio visualizer. By applying the CHARMm force field, ligands were energetically minimized using the steepest descent algorithm for 500 steps at an RMS gradient energy of $0.01 \mathrm{kcal} / \mathrm{mol}$ [21]. 


\subsection{Drug-likeness and ADMET prediction.}

Lipinski rule of five (RO5) was employed to predict the drug-likeness of ligands. RO5 includes molecular mass $(<=500$ Dalton), high lipophilicity $(\log \mathrm{p}<=5) \mathrm{H}$-bond donors $(<$ $=5)$, and H-bond acceptors $(<=10)$. These filtrations ensure drug-likeness for molecules obeying two or more features of RO5 [22,23]. Absorption, distribution, metabolism, excretion, and toxicity properties of all ligands were predicted using the PreADMET server (http://preadmet.bmdrc.org/) [24,25].

\subsection{Computational simulation and binding affinity prediction.}

ZDOCK and DockThor tool was used for protein-protein and protein-protein complex and ligand interactions, respectively [26,27]. The shape complementarity, desolvation, and electrostatic energy in ZDOCK generate the top 10 docking models. DockThor tool is based on multiple solution genetic algorithms and employs the MMFF94S scoring function to predict docked molds' best pose [28].

The PROtein binDIng enerGY (PRODIGY) server was used to analyze the molecular interaction tendency and stability in terms of binding affinity $(\Delta \mathrm{G})$ and dissociation constant (Kd) $[29,30]$. Docked complexes having the least RMSD and greater binding affinity were taken forward for further docking analysis.

\subsection{Visualization of docked complexes.}

The surface structure and interacting residues of docked complexes were visualized using CHIMERA, developed at the University of California, San Francisco (UCSF), USA, and DIMPLOT of LigPlot+, respectively [31,32]. The DIMPLOT uses 3D coordinates of interacting molecules and generates $2 \mathrm{D}$ schematic diagrams wherein dashed lines and arcs exhibiting $\mathrm{H}$-bonds and hydrophobic interactions, respectively.

\subsection{Molecular dynamics simulation.}

Stability and conformational changes of HSPs and ligands complexes were checked through the MD simulation (MDS) process of $30 \mathrm{~ns}$. Based on the docking score and the interaction analysis, compounds were chosen for energy minimization and MDS studies using the Desmond molecular dynamics system 3.1 with the OPLS AA force field. Water molecules were placed using the Desmond system builder by soaking the course with a pre-equilibrated TIP3P model from the orthorhombic water box along with $0.15 \mathrm{Na}+\mathrm{Cl}$ - in the system as neutralizing compounds [33]. Minimization of the prepared system was performed up to a maximum of 3000 steps using the steepest descent until a gradient threshold of $25 \mathrm{kcal} / \mathrm{mol} / \AA$. During the MDS, the smooth Particle-Mesh-Ewald method was used to calculate long-range electrostatic interactions [34]. $9 \AA$ cutoff radius value was used for the Coulumbic short-range interaction cutoff method. Frames of trajectory were captured at each 4.8 ps time step. Root mean square deviation (RMSD) and root mean square fluctuation (RMSF) of the backbone were calculated through the simulation with the first frames as a reference [35].

\subsection{CYP450 metabolism prediction.}

We predicted all probable metabolism sites for CYP3A4, CYP2D6, and CYP2C9 to explain selected ligands' metabolism using SMARTCyp 3.0. tool $[25,36]$. 


\subsection{Reactivity site prediction.}

Sites of reactivity and molecular reactivity of selected ligands were predicted using the XenoSite server (http://swami.wustl.edu/xenosite/). It depicts in vivo metabolism and small chemical compounds' reactivity based on Xenosite CYP P450 prediction models [25,37].

\subsection{Biological activity spectrum identification}

PASS online server (http://www.pharmaexpert.ru/passonline/) was used to predict the ligands interactions with different biological attributes using $\mathrm{Pa}$ and $\mathrm{Pi}$ representing to be found in a subclass of active and inactive compounds, respectively. The value of $\mathrm{Pa}$ and $\mathrm{Pi}$ lies from 0.000 to $1.000[25,38]$.

\section{Results and Discussion}

\subsection{Drug-likeness and ADMET prediction}

Drug-likeness filtration was carried out using Lipinski's RO5. Three violations in solanine and one violation in piperaquine, solanidine, spergaulin, geldanamycin, and Ver155008 were observed. The reference value for molecular weight (MW), hydrogen bond donor (HBD), hydrogen bond acceptor (HBA), and $\operatorname{LogP}$ is $\leq 500 \mathrm{Da}, \leq 5, \leq 10$, and $\leq 5$, respectively. RO5 values of compounds are shown in Table 1.

The ADME and toxicity of selected molecules were predicted. Human intestinal absorption (HIA), blood-brain barrier (BBB), plasma protein binding (ppb), Madin-Darby canine kidney cell permeability (MDCK), and heterogeneous human epithelial colorectal adenocarcinoma cell permeability (Caco2) were taken under consideration. Non-toxic molecules were filtered using mutagenicity and carcinogenicity mouse and rat models. ADMET values of compounds are shown in Table 2.

Table 1. Drug-likeness prediction of ligands.

\begin{tabular}{l|l|l|l|l} 
Compounds & MW $(\mathbf{g} / \mathbf{m})$ & HBD & HBA & LogP \\
\hline Aspirin & 180.16 & 1 & 4 & 1.24 \\
\hline Chloroquine & 319.9 & 1 & 3 & 4.04 \\
\hline Cinchonidine & 294.4 & 1 & 3 & 3.16 \\
\hline Cinchonine & 294.4 & 1 & 3 & 3.16 \\
\hline Erysolin & 285.3 & 2 & 4 & 1.48 \\
\hline Hydroxychloroquine & 335.9 & 2 & 4 & 3.01 \\
\hline Piperaquine & 535.5 & 0 & 6 & 3.84 \\
\hline Quinidine & 324.4 & 1 & 4 & 3.17 \\
\hline Quinine & 324.4 & 1 & 4 & 3.17 \\
\hline Sarcophine & 316.4 & 0 & 3 & 4.63 \\
\hline Solanidine & 397.6 & 1 & 2 & 5.65 \\
\hline Solanine & 868.1 & 9 & 16 & 0.00 \\
\hline Spergualin & 403.5 & 8 & 7 & -2.55 \\
\hline Geldanamycin & 560.6 & 3 & 9 & 0.00 \\
\hline Ver155008 & 556.4 & 4 & 10 & -0.21
\end{tabular}

Table 2. ADMET prediction of ligands.

\begin{tabular}{|c|c|c|c|c|c|c|c|c|}
\hline \multirow[b]{2}{*}{ Compounds } & \multirow{2}{*}{${ }^{\#} \mathrm{HIA}$} & \multirow{2}{*}{$\$ \mathbf{B B B}$} & & \multirow[b]{2}{*}{$\begin{array}{l}\text { Carcinogenicity } \\
\text { (Rat) }\end{array}$} \\
\hline & & & ${ }^{\circledR} \mathbf{P P B}$ & $\begin{array}{l}{ }^{\&} \text { MDCK cell } \\
\text { permeability }\end{array}$ & $\begin{array}{l}{ }^{*} \text { Caco2 cell } \\
\text { permeability }\end{array}$ & $\begin{array}{l}\text { Ames } \\
\text { test }\end{array}$ & $\begin{array}{l}\text { Carcinogenicity } \\
\text { (Mouse) }\end{array}$ & \\
\hline Aspirin & 90.18 & 0.72 & 37.42 & 37.95 & 20.09 & mutagen & negative & positive \\
\hline Chloroquine & 92.77 & 4.37 & 57.35 & 1.52 & 23.01 & $\begin{array}{l}\text { non- } \\
\text { mutagen }\end{array}$ & negative & positive \\
\hline Cinchonidine & 92.22 & 3.75 & 14.71 & 273.79 & 51.18 & mutagen & negative & negative \\
\hline ht & ce & 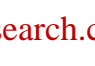 & & & & & & 12368 \\
\hline
\end{tabular}




\begin{tabular}{|c|c|c|c|c|c|c|c|c|}
\hline Compounds & ${ }^{\#}$ HIA & ${ }^{\$ \mathbf{B B B}}$ & ${ }^{@} \mathbf{P P B}$ & $\begin{array}{l}{ }^{\&} \text { MDCK cell } \\
\text { permeability }\end{array}$ & $\begin{array}{l}\text { "Caco2 cell } \\
\text { permeability }\end{array}$ & $\begin{array}{l}\text { Ames } \\
\text { test }\end{array}$ & $\begin{array}{l}\text { Carcinogenicity } \\
\text { (Mouse) }\end{array}$ & $\begin{array}{l}\text { Carcinogenicity } \\
\text { (Rat) }\end{array}$ \\
\hline Cinchonine & 92.22 & 3.75 & 14.71 & 273.79 & 51.18 & mutagen & negative & negative \\
\hline Erysolin & 90.96 & 0.76 & 30.79 & 44.14 & 25.05 & $\begin{array}{l}\text { non- } \\
\text { mutagen }\end{array}$ & negative & negative \\
\hline Hydroxychloroquine & 88.29 & 1.00 & 29.94 & 50.91 & 50.37 & mutagen & negative & negative \\
\hline Piperaquine & 95.07 & 2.86 & 11.01 & 10.90 & 26.61 & mutagen & negative & positive \\
\hline Quinidine & 92.29 & 1.26 & 11.37 & 33.85 & 47.99 & mutagen & negative & negative \\
\hline Quinine & 92.29 & 1.26 & 11.37 & 33.85 & 47.99 & mutagen & negative & negative \\
\hline Sarcophine & 95.18 & 6.47 & 100.00 & 69.58 & 49.40 & $\begin{array}{l}\text { non- } \\
\text { mutagen }\end{array}$ & negative & negative \\
\hline Solanidine & 99.86 & 13.22 & 97.92 & 4.17 & 55.23 & mutagen & negative & positive \\
\hline Solanine & 20.77 & 0.02 & 39.29 & 0.04 & 20.17 & $\begin{array}{l}\text { non- } \\
\text { mutagen }\end{array}$ & negative & negative \\
\hline Spergualin & 4.25 & 0.029 & 36.89 & 0.53 & 15.32 & mutagen & negative & negative \\
\hline Geldanamycin & 20.31 & 0.06 & 12.84 & 0.04 & 20.31 & $\begin{array}{l}\text { non- } \\
\text { mutagen }\end{array}$ & negative & negative \\
\hline Ver155008 & 92.76 & 0.02 & 84.59 & 0.04 & 19.86 & mutagen & negative & positive \\
\hline $\begin{array}{l}{ }^{\#} \text { HIA: Hum } \\
{ }^{\$} \text { BBB: Bloo } \\
\text { @ PPB: Plasr } \\
\text { \&MDCK: M } \\
{ }^{*} \text { Caco2: Het } \\
>70)\end{array}$ & $\begin{array}{l}\text { ntestina } \\
\text { rain bar } \\
\text { protein } \\
\text { n-Darby } \\
\text { geneous }\end{array}$ & $\begin{array}{l}\text { absorpt } \\
\text { er (CNS } \\
\text { inding } \\
\text { canine } 1 \\
\text { human }\end{array}$ & $\begin{array}{l}\text { (Activi } \\
\text { ctive cor } \\
\text { hemicals } \\
\text { lney cell } \\
\text { thelial c }\end{array}$ & $\begin{array}{l}\text { range: Poorly- }( \\
\text { pounds }(+):>1,( \\
\text { trongly bound }> \\
\text { ermeability (Lov } \\
\text { orectal adenocar }\end{array}$ & $\begin{array}{l}20 \% \text {, Moderate } \\
\text { NS inactive com } \\
0 \%, \text { Chemicals } \\
\text { r- }<25 \text {, Moder } \\
\text { noma cell perm }\end{array}$ & $\begin{array}{l}0 \sim 70 \%, \mathrm{Hi} \\
\text { unds (-): }< \\
\text { akly boun } \\
25 \sim 500, \mathrm{I} \\
\text { oility (Low }\end{array}$ & $\begin{array}{l}-70 \sim 100 \%) \\
\text { gher- > 500) } \\
-<4, \text { Moderate- } 4 \text { - }\end{array}$ & , Higher- \\
\hline
\end{tabular}

\subsection{Docking simulation and binding affinity analysis.}

\subsubsection{S-protein and ACE2.}

The molecular docking of viral S-protein (S) and cellular ACE2 protein was carried out using the ZDOCK tool [26]. The plausible molecular interaction was depicted between S-and ACE2 protein, as highlighted by its Z-dock score 1457. Further, docking stability of the SACE2 was checked in terms of $\Delta \mathrm{G}\left(\mathrm{kcal} \mathrm{mol}^{-1}\right)$ and $\mathrm{K}_{\mathrm{d}}$ that was found $-18.7 \mathrm{kcal} \mathrm{mol}^{-1}$ and $1.5 \times 10^{-15} \mathrm{M}$, respectively [29,30]. Residues Q493, K417, Y453, T376, Y505, and R403 of Sprotein was involved in H-bonding with Y255, S254, P612, D136, and D157 residues of ACE2. R403 residue of S-protein also exhibited salt bridge bonding with D157 residue of ACE2. Further, A475, G476, N487, F456, Y473, N489, L455, E484, F486, F490, Y505, Y449, G496, Q498, T500, N501, L455, and Y502 residues of S-protein was involved in hydrophobic interaction with Q24, S19, T27, F28, Y83, D30, K31, H34, A36, L79, M82, R393, G354, K353, E37, D38, Q42, Y41, L45, D355, R357, and N330 residues of ACE2. These interacting residues are in agreement with the reported data earlier [3].

\subsubsection{HSP70 and S-ACE2 complex.}

Docking simulation of HSP70 and S-ACE2 complex exhibited Z-dock score 1598. $\Delta \mathrm{G}$ and $\mathrm{K}_{\mathrm{d}}$ value of HSP70-S-ACE2 was found $-17.0 \mathrm{kcal} \mathrm{mol}^{-1}$ and $3.3 \times 10^{-13} \mathrm{M}$, respectively. Residues N322, K309, M323, and Q325 of ACE2 were involved in H-bonding with V169, G166, N168, and A4 residues HSP70. K313 and E325 residues of ACE2 also exhibited salt bridge bonding with D160 and K3 residues of HSP70. Moreover, the residues P412, K378, C379, A435, Y369, and T385 of S-protein was involved in H-bonding with K190, D214, R187, G184, and R193 residues of HSP70. Hydrophobic residues involved in molecular interactions at the interface are shown in Table 3. 
Table 3. Hydrophobic residues at the interface of the HSP70-S-ACE2 complex.

\begin{tabular}{l|l|l} 
HSP70 & S-protein & ACE2 \\
\hline G215, I379, T188, G189, H23, L185, & V503, Y380, I434, S375, T374, F377, & N330, W328, V316, E312, \\
$\begin{array}{l}\text { Y183, V26, G24, F21, D332, K25, } \\
\text { Y371, V375, L170, L167, K159, V163, }\end{array}$ & F374, P384, S373, A372, S383, S371 & Q305 \\
T140, N141, A5 & & \\
\hline HSP90 & S-protein & \\
\hline F213, Q212, G215, I214, P217, Y216, & P479, F486, N481, G485, V483, F490, & V107, L79, M82, T78, E75, \\
L64, T219, I218, F221, V207, K204 & G482 & L39, F72, E35
\end{tabular}

\subsubsection{HSP70 and AngII.}

HSP70-AngII complex depicted a Z-dock score of 1326. $\Delta \mathrm{G}$ and $\mathrm{K}_{\mathrm{d}}$ value of the complex was found $-12.0 \mathrm{kcal} \mathrm{mol}^{-1}$ and $1.6 \times 10^{-9} \mathrm{M}$, respectively. R272, T204, R342, T14, G202, S340, and K271 residues of HSP70 were engaged in H-bonding with D1, V3, F8, H6, Y4, and P7 residues of AngII. H6, R2, and D1 residues of AngII also exhibited salt bridge bonding with E268, R272, E367, and R269 residues of HSP70. Moreover, D199, N364, Y15, G368, T13, S276, G230, S275, I343, T37, F233, L200, G201, and G203 residues of HSP70 was also involved in hydrophobic interactions.

\subsubsection{HSP90 and S-ACE2 complex.}

Docking simulation of HSP90 and S-ACE2 complex exhibited a Z-dock score of 1648. $\triangle \mathrm{G}$ and $\mathrm{K}_{\mathrm{d}}$ value of HSP90-S-ACE2 was found $-12.9 \mathrm{kcal} \mathrm{mol}^{-1}$ and $3.7 \times 10^{-10} \mathrm{M}$, respectively. Residues S106 and Q81 of ACE2 were involved in H-bonding with Y61 and R60 residues HSP90. E110 and K74 residues of ACE2 also exhibited salt bridge bonding with K58 and D57 residues of HSP90. Moreover, the residues C480 and E484 of S-protein were involved in Hbonding and salt bridge bonding with L220 and K208 residues of HSP90. Hydrophobic residues involved in molecular interactions at the interface are shown in Table 3.

\subsubsection{HSP90 and AngII.}

HSP90-AngII complex depicted a Z-dock score of 1385. $\Delta \mathrm{G}$ and $\mathrm{K}_{\mathrm{d}}$ value of the complex was found $-9.1 \mathrm{kcal} \mathrm{mol}^{-1}$ and $2.0 \times 10^{-7} \mathrm{M}$, respectively. N106, K112, and N51 residues of HSP90 were engaged in $\mathrm{H}$-bonding with $\mathrm{I} 5, \mathrm{~F} 8$, and Y4 residues of AngII. Moreover, S113, V136, A111, I210, G108, N105, I26, G135, L107, F138, G137, V186, T184, S52, L48, D93, and D54 residues of HSP90 was also involved in hydrophobic interactions.

\subsubsection{HSP70-S-ACE2 complex and Ligands.}

Docking simulation of HSP70-S-ACE2 complex and selected ligands was carried out using the DockThor tool [27]. Aspirin (ASP), Piperaquine (PIP), and Hydroxychloroquine (HYD) exhibited better interactions as compared to other ligands having a DockThor score of $-7.50,-8.74$, and -7.32, respectively. Moreover, K3 residue of HSP70, G502, V503, and G502 residues of S-protein, M383, P321, M323, T324, Q325, and G354 residues of ACE2 were found in close vicinity to ASP. Similarly, K3 of HSP70, T500 of S-protein, W48, N49, Q305, W328, E329, N330, S331, M332, L333, T334, and R357 residues of ACE2 were found in close contact to PIP. Likewise, I379 of HSP70, D405, G502, V503, and G504 of S-protein, A386, G354, and A387 residues of ACE2 were found in surrounding HYD. Docking stability $(\Delta \mathrm{G}$ and $\mathrm{K}_{\mathrm{d}}$ ) of HSP70-S-ACE2-ASP/PIP/HYD is shown in Table 4. 
3.2.7. HSP70-AngII complex and Ligands.

Ligands ASP, PIP, and HYD also depicted better binding than the remaining ligands having a DockThor score of -8.15, -9.40, and -7.95, respectively. Moreover, T37, P39, D53, K56, N57, and E268 residues of HSP70, D1, and I5 residues of AngII exhibited close contact with ASP. Similarly, Y15, N35, R36, T37, K56, D268, R269, D366, R272, and E367 residues of HSP70, D1, and I5 residues of AngII were found in close vicinity to PIP. Likewise, G34, N35, T37, E268, N364, and D366 residues of HSP70, D1, R2, and I5 residues of AngII were engaged in surrounding HYD. $\Delta \mathrm{G}$ and Kd values of HSP70-AngII-ASP/PIP/HYD are shown in Table 4. Various residues of the complexities involved in molecular interactions are shown in Figure 1.

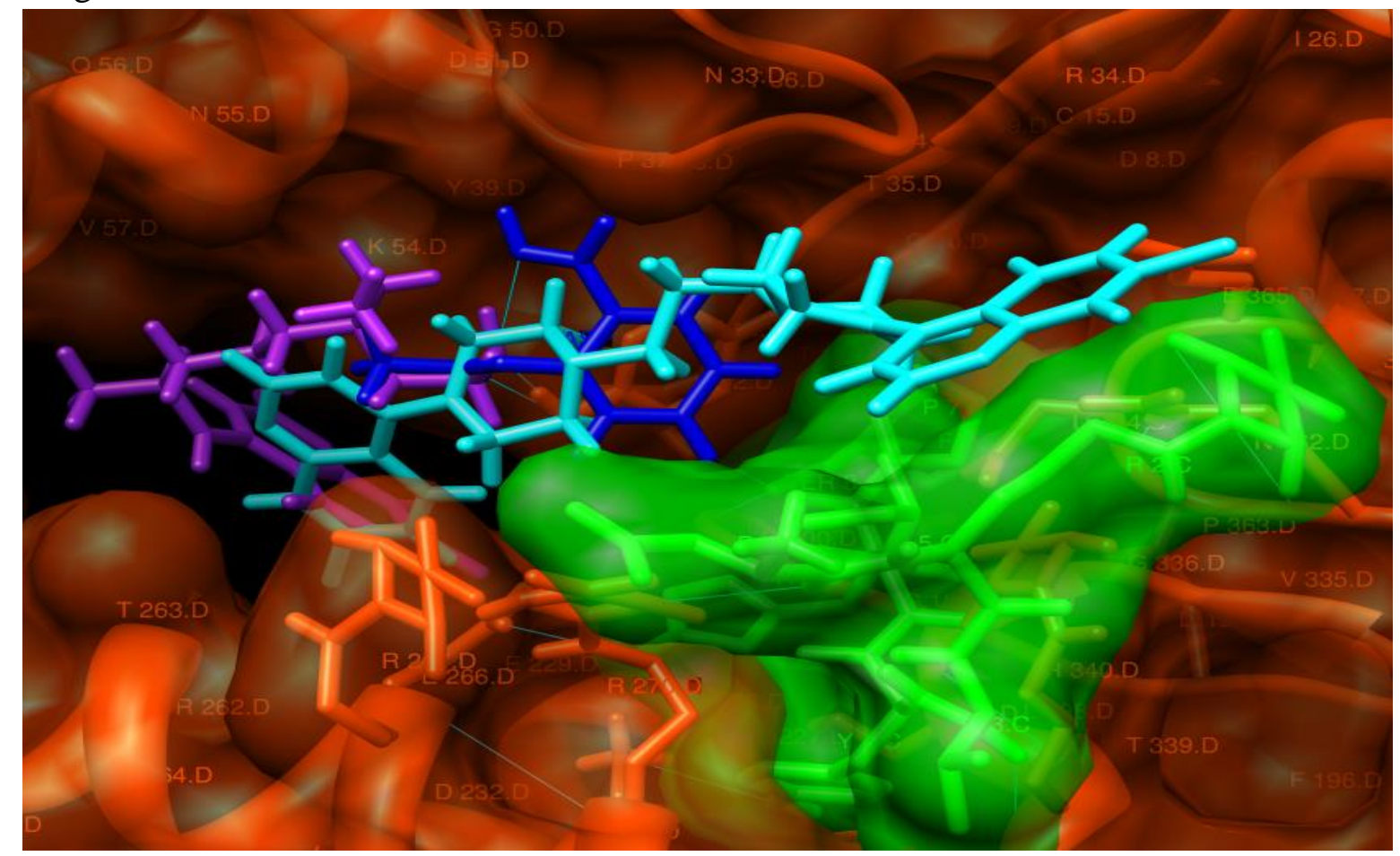

Figure 1. Docked complex of HSP70-AngII-ASP/PIP/HYD. HSP70, AngII, ASP, PIP, and HYD are shown in orange, green, blue, cyan, and purple respectively.

\subsubsection{HSP90-S-ACE2 complex and Ligands.}

Similar to the HSP70-S-ACE2 and HSP70-AngII complexes, ligands ASP (DockThor score: -7.30), PIP (-8.22), and HYD (-7.28) also showed better interactions than other ligands. Moreover, K74, V92, Y142, and I218 residue of HSP90, G471, T430, and N388, residues of S-protein, G104, W303, N117, H378, and D382 residues of ACE2 were engaged in close contact to ASP. Similarly, Y61, R60, and K74 residues of HSP90, C480, E471, and N354 of S-protein, L100, Q102, N103, G104, S106, N117, S113, Y202, and W303 residues of ACE2 exhibited close contact with PIP. Likewise, A166, D175, K208, and V222 of HSP90, R346, S75, R410, and T430 residues of S-protein, K187, Y199, D206, Y202, D509, Y510, and S511 residues of ACE2 were found in surrounding HYD. $\triangle \mathrm{G}$ and Kd values of HSP90-S-ACE2ASP/PIP/HYD are shown in Table 4.

\subsubsection{HSP90-AngII complex and Ligands.}

Ligands ASP, PIP, and HYD also exhibited better binding than the remaining ligands having a DockThor score of -7.89, -9.19, and -7.58, respectively. Moreover, K147, T149, I151, 
A161, E163, R173, D175, G177, K185, I187, and H189 residues HSP90, F8, P7, and H6 residues of AngII exhibited close contact with ASP. Similarly, I81, D86, T90, V102, I151, E178, M180, G181, R182, K185, and I187 residues of HSP90, F8, I5, and V3 residues of AngII were found in close vicinity to PIP. Likewise, K147, T149, I151, A161, E163, R173, D175, G177, K185, and H189 residues of HSP90, I5, H6, and F8, residues of AngII were engaged in surrounding HYD. $\Delta \mathrm{G}$ and Kd values of HSP90-AngII-ASP /PIP/HYD are shown in Table 4.

Table 4. Complex-ligand docking analysis.

\begin{tabular}{l|l|l|l|l} 
Molecular interaction & ${ }^{*} \mathbf{D o c k}$ Thor score & $\Delta \mathbf{G}^{*}\left(\mathbf{k c a l ~}_{\mathbf{~ m o l}}{ }^{-\mathbf{1}}\right)$ & ${ }^{*} \mathbf{K}_{\mathbf{d}}(\mathbf{M})$ & $\mathbf{H}$-bonding \\
\hline HSP70-S-ACE2-ASP & -7.50 & -15.2 & $2.7 \times 10^{-11}$ & 4 \\
\hline HSP70-S-ACE2-PIP & -8.74 & -16.7 & $3.2 \times 10^{-12}$ & 5 \\
\hline HSP70-S-ACE2-HYD & -7.32 & -15.8 & $3.3 \times 10^{-11}$ & 4 \\
\hline HSP70-AngII-ASP & -8.15 & -19.5 & $3.2 \times 10^{-15}$ & 7 \\
\hline HSP70-AngII-PIP & -9.40 & -20.3 & $3.4 \times 10^{-16}$ & 8 \\
\hline HSP70-AngII-HYD & -7.95 & -18.5 & $1.2 \times 10^{-14}$ & 7 \\
\hline HSP90-S-ACE2-ASP & -7.30 & -11.9 & $2.8 \times 10^{-9}$ & 3 \\
\hline HSP90-S-ACE2-PIP & -8.22 & -12.4 & $5.4 \times 10^{-9}$ & 3 \\
\hline HSP70-S-ACE2-HYD & -7.28 & -10.0 & $3.9 \times 10^{-8}$ & 3 \\
\hline HSP90-AngII-ASP & -7.89 & -19.2 & $1.3 \times 10^{-15}$ & 4 \\
\hline HSP90-AngII-PIP & -9.19 & -20.6 & $5.5 \times 10^{-16}$ & 4 \\
\hline HSP90-AngII-HYD & -7.58 & -14.8 & $1.5 \times 10^{-11}$ & 4
\end{tabular}

\#DockThor score: Scoring function of DockThor tool. The higher score signifies a stronger binding [27].

*Binding affinity $(\Delta G)$ and dissociation constant $\left(\mathrm{K}_{\mathrm{d}}\right)$ predicted by PRODIGY) server [30].

\subsection{MD simulation analysis.}

The Ligands ASP, PIP, and HYD showed comparatively better interaction with HSP70AngII. Consequently, the explicit solvent MD simulations of these complexes for $30 \mathrm{~ns}$ were performed to envisage conformational stability. RMSD graph of the protein-ligands complex reveals that the overall RMSD is around 1-3.5 $\AA$.
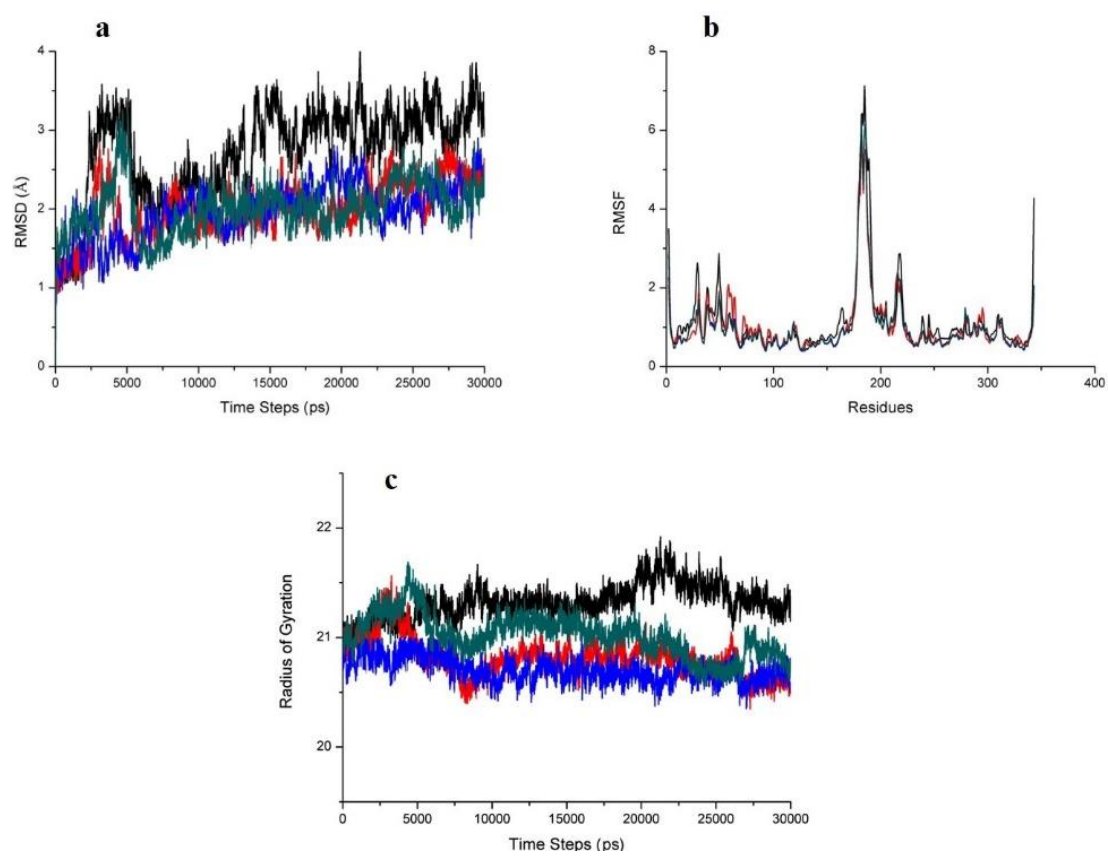

Figure 2. MD simulation of ligands binding to the HSP70-AngII (a) RMSD plot as a function of time. Black, red, blue, and grey colors represent Ver155008, ASP, PIP, and HYD, respectively. (b) RMSF plot for Ver155008 (black), ASP (red), PIP (blue), and HYD (grey) (c) Time evolution of Rg values of ASP (red), PIP (blue), and HYD (grey) during $30 \mathrm{~ns}$ MD simulation. 
The overall graph states that the RMSD of the protein with the ligand PIP shows stability compared to other complexes. The RMSF graph states that residues 180 to 200 got fluctuated. Rg states that the compactness of the protein-ligand complexes is maintained throughout the analysis. The root mean square deviation (RMSD), root mean square fluctuation of residues (RMSF), and radius of gyration $(\mathrm{Rg})$ are shown in Figure 2a-c.

\subsection{CYP450 metabolism prediction of selected ligands.}

CYP450 metabolism of ASP, PIP, and HYD was compared with the known inhibitor of HSP70 (Ver155008) based on score, energy, COO-dist, Span2end, and 2D-SASA [39]. The most probable CYP3A4 site of metabolism and their attributes predictions are shown in Figure 3a-d and Table 5, respectively.

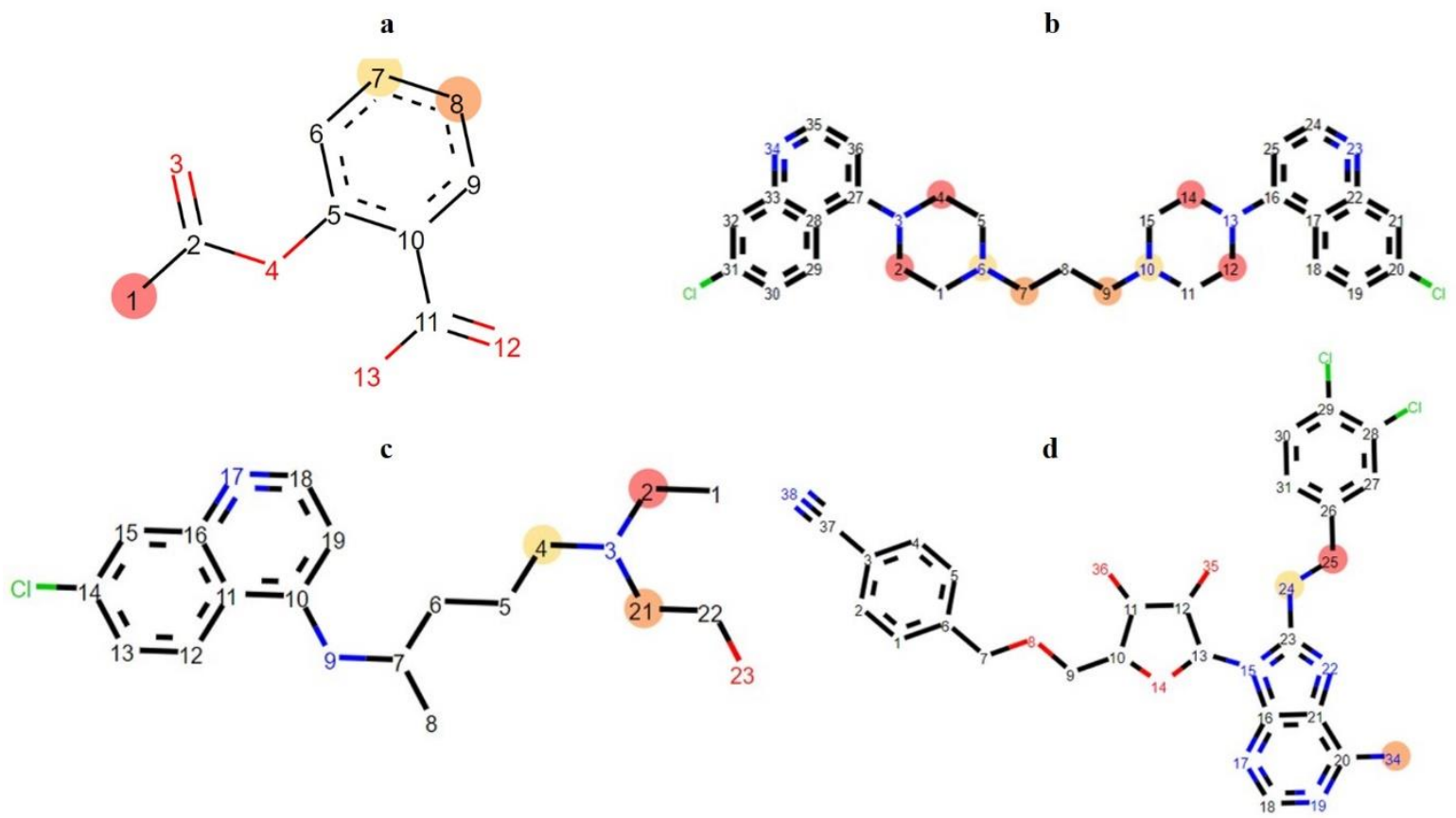

Figure 3. CYP3A4 site metabolism prediction (a) ASP, (b) PIP, (c) HYD, (d) Ver155008.

Table 5. SMARTCyp prediction score for 3A4.

\begin{tabular}{|c|c|c|c|c|c|c|c|c|}
\hline Compounds & $\begin{array}{l}3 \mathrm{~A} 4 \\
\text { ranking }\end{array}$ & Atom & $\begin{array}{l}\text { 3A4 } \\
\text { score }\end{array}$ & ${ }^{\#}$ Energy & $\begin{array}{l}\text { 2D } \\
\text { SASA }\end{array}$ & Span2end & $\begin{array}{l}\text { Relative } \\
\text { span }\end{array}$ & Similarity \\
\hline \multirow[t]{3}{*}{ ASP } & 1 & C. 1 & 56.0 & 66.4 & 59.3 & 0 & 1.0 & 0.7 \\
\hline & 2 & C. 8 & 71.5 & 80.8 & 32.7 & 0 & 1.0 & 1.0 \\
\hline & 3 & C.7 & 72.8 & 80.8 & 33.5 & 1 & 0.8 & 1.0 \\
\hline \multirow[t]{3}{*}{ PIP } & 1 & C. 2 & 34.5 & 41.1 & 27.6 & 7 & 0.7 & 0.7 \\
\hline & 2 & C.7 & 35.8 & 41.1 & 23.3 & 10 & 0.5 & 0.4 \\
\hline & 3 & N.6 & 37.7 & 42.6 & 3.9 & 9 & 0.6 & 0.7 \\
\hline \multirow[t]{3}{*}{ HYD } & 1 & C. 2 & 33.1 & 41.1 & 27.9 & 2 & 0.9 & 0.7 \\
\hline & 2 & C.21 & 33.2 & 41.1 & 25.7 & 2 & 0.9 & 0.4 \\
\hline & 3 & C.4 & 34.5 & 41.1 & 21.0 & 4 & 0.7 & 0.4 \\
\hline \multirow[t]{3}{*}{ Ver155008 } & 1 & C. 25 & 34.1 & 41.1 & 23.8 & 5 & 0.7 & 0.4 \\
\hline & 2 & N.34 & 45.8 & 54.1 & 47.0 & 4 & 0.7 & 0.7 \\
\hline & 3 & N.24 & 48.1 & 54.1 & 10.5 & 6 & 0.6 & 0.3 \\
\hline
\end{tabular}

\#Reference values: Energy (<999), span2end (<=4), relative span (0.5-1), similarity (0-1) [39].

\subsection{Reactivity site prediction of selected ligands.}

XenoSite CYP P450 prediction models reveal the metabolically labile positions of an atom within potential substrates, e.g., DNA, protein, glutathione, and cyanide, epoxide, and 
quinone. The value 1 and 0 signifies reactivity and non-reactivity, respectively [40]. The metabolically labile positional value of selected ligands was found between the specified range.

\subsection{Biological activity spectrum identification.}

PASS algorithm forecasts numerous biological activity of small chemical compounds using their structural features, thereby minimize the attrition rate before their in vitro and in vivo experimentations. Selected ligands exhibited better antiviral, anti-neurogenic, antineoplastic, and cardio-protectant activity than known inhibitor Ver155008. It showed a value of $\mathrm{Pa}$ and $\mathrm{Pi}$ within the specified range [25,38].

Patients suffering from cardiac, metabolic, pulmonary, and degenerative disorders exhibited impaired heat shock responses (HSRs) triggered by the oxidative, metabolic, and thermal stresses. Individuals showed such morbidities when infected with COVID-19 exasperated pre-existing jeopardized HSRs resulting in low expression of HSPs. Data accumulated so far showed the engagement of HSPs, especially HSP70 and HSP90, in SARSCoV-2 -mediated cytokine storm inhibition via NFKB and interferon (IFN)-mediated pathways $[18,41]$. So, people with malfunctioned HSR mechanisms step down virus-mediated inflammatory burst, subsequently pave the way for ARDS. Cytokine-induced ARDS phenomenon perpetually happens in some bat species, considered the natural host of zoonotic viruses, including SARS-CoV-2. Still, it does not get infected or forecast any disease symptoms due to better evolutionary adopted HSR mechanisms contrary to humans. Moreover, accumulated data highlights sodium salicylate and aspirin's utility as an inducer of HSPs [41].

The $\Delta \mathrm{G}$ and $\mathrm{K}_{\mathrm{i}}$ have been considered a standard parameter for assessing interaction potential at the in silico platform. The efficacy of preferred inhibition is competitive and is governed by the shape complementarily and Binding energy. Binding energy denotes the amount of energy released when two molecules interact to find a suitable complex, and as per laws of thermodynamics, the more the energy released more stable the bound complex will be. Henceforth, the molecular docking studies are always searching for protein complexes with higher $\Delta \mathrm{G}$ and lower $\mathrm{K}_{\mathrm{i}}$ value to find more stable complexes to function [42-45]. In the purview of the above findings, we have proposed a hypothetical pathway of virus entry into the host cell via S-protein and ACE2 interaction and the most probable underlying protective mechanism of HSPs through protein-protein and ligands' interactions (Figure 4).

In a typical cell, the conversion of AngII into AngI-VII is facilitated by ACE2, thus putting its normal impact over other biological systems. On the other hand, in COVID-19 patients, the jeopardized ACE2 mechanism leads to the accumulation of AngII, consequently induced inflammatory pathways accelerate the cytokine storm causing ARDS. In the proposed mechanism, activated HSPs (HSP70 and HSP90) have two proteins for molecular interactions: the S-ACE2 complex and AngII. The stability of complex formation were found as followsHSP70-S-ACE2 ( $\triangle \mathrm{G}$ : $-17.0 \mathrm{kcal} \mathrm{mol}^{-1}$; Kd: $\left.3.3 \times 10^{-13} \mathrm{M}\right)>$ HSP90-S-ACE2 (-12.9 kcal mol ${ }^{-1}$; $\left.3.7 \times 10^{-10} \mathrm{M}\right)>$ HSP70-AngII $\left(-12.0 \mathrm{kcal} \mathrm{mol}^{-1} ; 1.6 \times 10^{-9} \mathrm{M}\right)>\mathrm{HSP} 90-$ AngII $\left(9.1 \mathrm{kcal} \mathrm{mol}^{-1}\right.$; $\left.2.0 \times 10^{-7} \mathrm{M}\right)$. Further, we have checked the top three ligands' molecular interaction, namely ASP, PIP, and HYD with HSP70-S-ACE2, HSP90-S-ACE2, HSP70-AngII, and HSP90-AngII complexes as they depicted better interaction with S-ACE2 complex in comparison to other ligands. Interestingly, HSP70-AngII-ASP/PIP/HYD's stability was found greater than HSP70S-ACE2-ASP/PIP/HYD. Similarly, HSP90-AngII-ASP/PIP/HYD's stability was found greater than HSP90-S-ACE2- ASP/PIP/HYD (Table 4). 


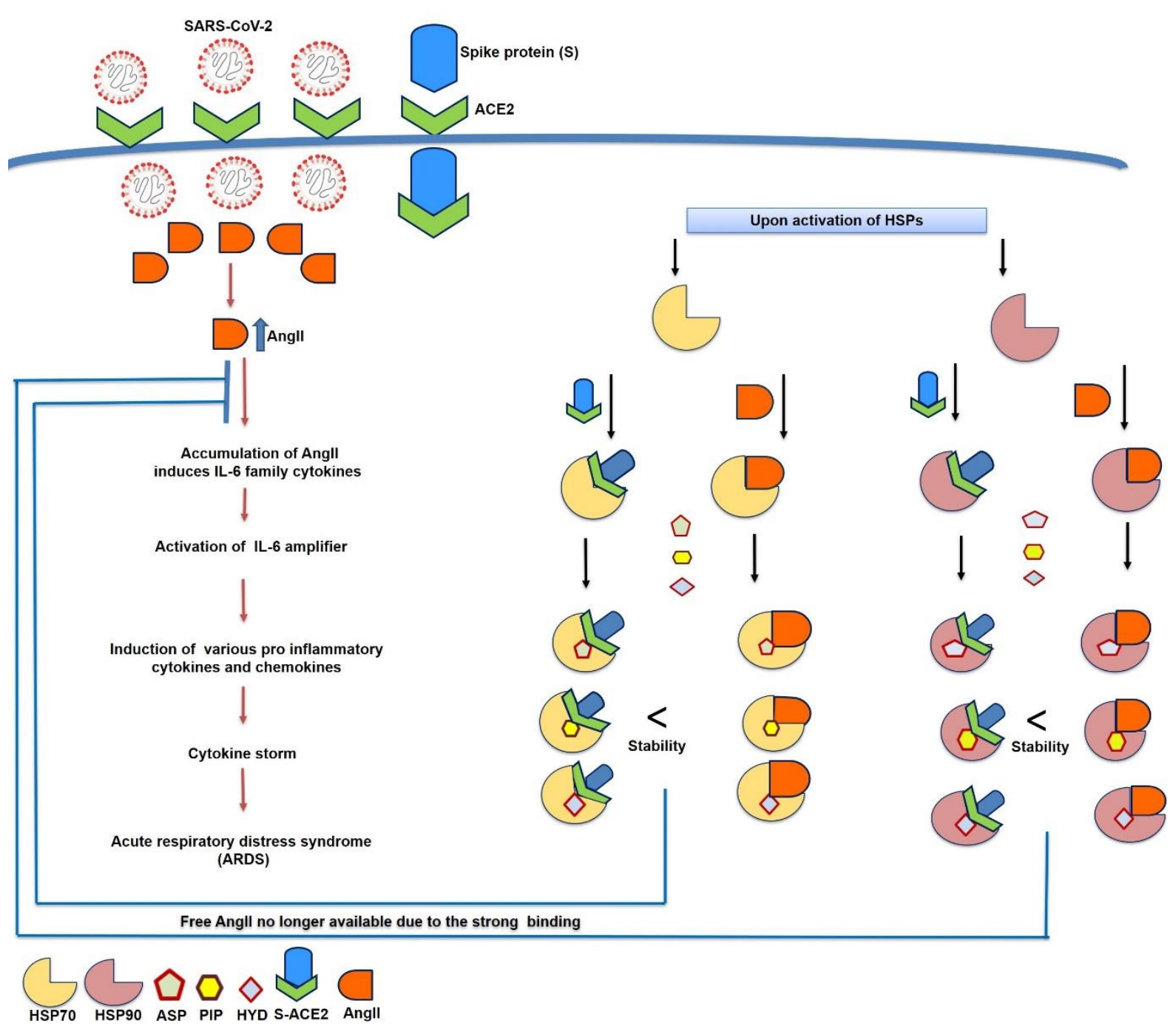

Figure 4. Hypothetical protective mechanism of HSPs in SARS-CoV-2 mediated ARDS.

The stability of HSP70-AngII-ASP/PIP/HYD complexes was confirmed by MD simulation analysis. It was concluded from MD simulation findings that all complexes show considerable stability throughout the simulation. However, when compared with the other compounds, PIP is considerably more stable, followed by ASP and HYD during 30ns simulation. MD simulation analysis exhibited that complexes remained stable during the entire simulation process (Figure 1). Pharmacokinetic properties of ligands were checked in terms of drug-likeness and ADMET features. Ligands ASP, PIP, and HYD completely agree with Lipinski RO5 (Table 1). Further, ADMET properties were also found in a considerable range (Table 2).

About 50\% biotransformation of small chemical compounds is carried out with the help of the CYP3A4 enzyme. Identifying the specific site of metabolism of compounds might be a promising step in drug discovery and development. All descriptors, including score, energy, COO-dist, Span2end, and 2D-SASA associated with CYP metabolism for ligands HYD, PIP, and HYD, were found in the specified range (Table 5). Biological efficacy and safety of lead molecules is another essential concern of the drug discovery pipeline. We have calculated metabolically labile sites of selected ligands using the Xenosite CYP P450 prediction model and found their values within the reference range and comparatively better than a known inhibitor of HSP70. Likewise, PASS online tool was also used to identify a broad spectrum of biological activity of ligands. ASP, PIP, and HYD exhibited plausible biological activity in comparison to the known inhibitor Ver155008. HSP70/90-AngII complexes were tightly 
bound with the selected ligands compared to the HSP70/90-S-ACE2 complexes as highlighted by their DockThor scores, $\Delta \mathrm{G}$, and $\mathrm{Kd}$ values. Based on the above findings, it might be possible that free AngII no longer available to induce cytokine pathways and thus inhibiting ARDS in COVID-19 patients.

\section{Conclusions}

Detailed protein-protein (S-ACE2), complex protein-protein (HSP70/90-S-ACE2), and complex protein-ligands interactions (HSP70/90-S-ACE2-ASP/PIP/HYD) were carried out to establish the most probable protective mechanism of HSPs in SARS-CoV-2 mediated ARDS using molecular interactions, MD simulation, and CYP450 metabolism studies. Findings depicted that PIP and ASP interacted more efficiently with HSP70/90-AngII compared to HSP70/90-S-ACE2 and formed a relatively stable complex with excellent DockThor scores, $\Delta \mathrm{G}$, and Kd values. It suggests that ASP-induced HSPs and PIP may prevent cytokine storm by binding with the AngII, making it unavailable or less available, thereby enhancing the natural immunity against the SARS-CoV-2. Further, detailed residues analysis and its implication toward establishing structural insights will help understand the underlying safeguard mechanism of PIP/ASP-induced HSPs in COVID-19 patients. The study's findings are purely based on in silico predictions; therefore, wet-lab experimentations are required for validation.

\section{Funding}

This research was funded by the project \# COVID-1916 (University of Hail, Saudi Arabia).

\section{Acknowledgments}

The authors thank the Deanship of Scientific Research at the University of Haíl, Saudi Arabia for providing financial and technical help through the project number COVID-1916.

\section{Conflicts of Interest}

The authors declare no conflict of interest.

\section{References}

1. Sornette, D.; Mearns, E.; Schatz, M.; Wu, K.; Darcet, D. Interpreting, analysing and modelling COVID-19 mortality data. Nonlinear Dynamics 2020, 101, 1751-1776, https://doi.org/10.1007/s11071-020-05966-z.

2. Li, H.; Liu, S.-M.; Yu, X.-H.; Tang, S.-L.; Tang, C.-K. Coronavirus disease 2019 (COVID-19): current status and future perspectives. Int. J. Antimicrob. Agents 2020, 55, 105951, https://doi.org/10.1016/j.ijantimicag.2020.105951.

3. Wang, Q.; Zhang, Y.; Wu, L.; Niu, S.; Song, C.; Zhang, Z.; Lu, G.; Qiao, C.; Hu, Y.; Yuen, K.-Y. Structural and functional basis of SARS-CoV-2 entry by using human ACE2. Cell 2020, https://doi.org/10.1016/j.cell.2020.03.045.

4. Prompetchara, E.; Ketloy, C.; Palaga, T. Immune responses in COVID-19 and potential vaccines: Lessons learned from SARS and MERS epidemic. Asian Pac. J. Allergy Immunol. 2020, 38, 1-9, https://doi.org/10.12932/AP-200220-0772.

5. Le, T.T.; Andreadakis, Z.; Kumar, A.; Roman, R.G.; Tollefsen, S.; Saville, M.; Mayhew, S. The COVID-19 vaccine development landscape. Nat. Rev. Drug Discov. 2020, 19, 305-306, https://doi.org/10.1038/d41573020-00073-5.

6. Lurie, N.; Saville, M.; Hatchett, R.; Halton, J. Developing Covid-19 Vaccines at Pandemic Speed. New Engl. J. Med. 2020, 382, 1969-1973, https://doi.org/10.1056/NEJMp2005630. 
7. Patel, A.B.; Verma, A. COVID-19 and Angiotensin-Converting Enzyme Inhibitors and Angiotensin Receptor Blockers: What Is the Evidence? JAMA 2020, 323, 1769-1770, https://doi.org/10.1001/jama.2020.4812.

8. Zheng, Y.-Y.; Ma, Y.-T.; Zhang, J.-Y.; Xie, X. COVID-19 and the cardiovascular system. Nature Reviews Cardiology 2020, 17, 259-260, https://doi.org/10.1038/s41569-020-0360-5.

9. Zhou, P.; Yang, X.-L.; Wang, X.-G.; Hu, B.; Zhang, L.; Zhang, W.; Si, H.-R.; Zhu, Y.; Li, B.; Huang, C.-L.; Chen, H.-D.; Chen, J.; Luo, Y.; Guo, H.; Jiang, R.-D.; Liu, M.-Q.; Chen, Y.; Shen, X.-R.; Wang, X.; Zheng, X.-S.; Zhao, K.; Chen, Q.-J.; Deng, F.; Liu, L.-L.; Yan, B.; Zhan, F.-X.; Wang, Y.-Y.; Xiao, G.-F.; Shi, Z.L. A pneumonia outbreak associated with a new coronavirus of probable bat origin. Nature 2020, 579, 270273, https://doi.org/10.1038/s41586-020-2012-7.

10. Astuti, I.; Ysrafil. Severe Acute Respiratory Syndrome Coronavirus 2 (SARS-CoV-2): An overview of viral structure and host response. Diabetes \& Metabolic Syndrome: Clinical Research \& Reviews 2020, 14, 407412, https://doi.org/10.1016/j.dsx.2020.04.020.

11. Hoffmann, M.; Kleine-Weber, H.; Schroeder, S.; Krüger, N.; Herrler, T.; Erichsen, S.; Schiergens, T.S.; Herrler, G.; Wu, N.-H.; Nitsche, A. SARS-CoV-2 cell entry depends on ACE2 and TMPRSS2 and is blocked by a clinically proven protease inhibitor. Cell 2020, https://doi.org/10.1016/j.cell.2020.02.052.

12. Letko, M.; Seifert, S.N.; Olival, K.J.; Plowright, R.K.; Munster, V.J. Bat-borne virus diversity, spillover and emergence. Nature Reviews Microbiology 2020, 18, 461-471, https://doi.org/10.1038/s41579-020-0394-z.

13. Morens, D.M.; Fauci, A.S. Emerging pandemic diseases: How we got to COVID-19. Cell 2020, https://doi.org/10.1016/j.cell.2020.08.021.

14. Banerjee, A.; Baker, M.L.; Kulcsar, K.; Misra, V.; Plowright, R.; Mossman, K. Novel Insights Into Immune Systems of Bats. 2020, 11, https://doi.org/10.3389/fimmu.2020.00026.

15. Chionh, Y.T.; Cui, J.; Koh, J.; Mendenhall, I.H.; Ng, J.H.J.; Low, D.; Itahana, K.; Irving, A.T.; Wang, L.-F. High basal heat-shock protein expression in bats confers resistance to cellular heat/oxidative stress. Cell Stress Chaperones 2019, 24, 835-849, https://doi.org/10.1007/s12192-019-01013-y.

16. Wan, Q.; Song, D.; Li, H.; He, M.-1. Stress proteins: the biological functions in virus infection, present and challenges for target-based antiviral drug development. Signal Transduction and Targeted Therapy 2020, 5, 125, https://doi.org/10.1038/s41392-020-00233-4.

17. Koo, H.N.; Oh, S.Y.; Kang, K.; Moon, D.Y.; Do Kim, H.; Kang, H.S. Modulation of HSP70 and HSP90 expression by sodium salicylate and aspirin in fish cell line CHSE-214. Zool. Sci. 2000, 17, 1275-1282, https://doi.org/10.2108/zsj.17.1275.

18. Tanaka, T.; Shibazaki, A.; Ono, R.; Kaisho, T. HSP70 mediates degradation of the p65 subunit of nuclear factor $\kappa \mathrm{B}$ to inhibit inflammatory signaling. Science Signaling 2014, 7, ra119, https://doi.org/10.1126/scisignal.2005533.

19. Janardhan, V.; Janardhan, V.; Kalousek, V. COVID-19 as a Blood Clotting Disorder Masquerading as a Respiratory Illness: A Cerebrovascular Perspective and Therapeutic Implications for Stroke Thrombectomy. J. Neuroimaging 2020, 30, 555-561, https://doi.org/10.1111/jon.12770.

20. Chow, J.H.; Khanna, A.K.; Kethireddy, S.; Yamane, D.; Levine, A.; Jackson, A.M.; McCurdy, M.T.; Tabatabai, A.; Kumar, G.; Park, P.; Benjenk, I.; Menaker, J.; Ahmed, N.; Glidewell, E.; Presutto, E.; Cain, S.; Haridasa, N.; Field, W.; Fowler, J.G.; Trinh, D.; Johnson, K.N.; Kaur, A.; Lee, A.; Sebastian, K.; Ulrich, A.; Peña, S.; Carpenter, R.; Sudhakar, S.; Uppal, P.; Fedeles, B.T.; Sachs, A.; Dahbour, L.; Teeter, W.; Tanaka, K.; Galvagno, S.M.; Herr, D.L.; Scalea, T.M.; Mazzeffi, M.A. Aspirin Use is Associated with Decreased Mechanical Ventilation, ICU Admission, and In-Hospital Mortality in Hospitalized Patients with COVID-19. Anesth. Analg. 2020, Publish Ahead of Print, https://doi.org/10.1213/ANE.0000000000005292.

21. Brooks, B.R.; Brooks Iii, C.L.; Mackerell Jr, A.D.; Nilsson, L.; Petrella, R.J.; Roux, B.; Won, Y.; Archontis, G.; Bartels, C.; Boresch, S.; Caflisch, A.; Caves, L.; Cui, Q.; Dinner, A.R.; Feig, M.; Fischer, S.; Gao, J.; Hodoscek, M.; Im, W.; Kuczera, K.; Lazaridis, T.; Ma, J.; Ovchinnikov, V.; Paci, E.; Pastor, R.W.; Post, C.B.; Pu, J.Z.; Schaefer, M.; Tidor, B.; Venable, R.M.; Woodcock, H.L.; Wu, X.; Yang, W.; York, D.M.; Karplus, M. CHARMM: The biomolecular simulation program. J. Comput. Chem. 2009, 30, 1545-1614, https://doi.org/10.1002/jcc.21287.

22. Lipinski, C.A. Lead- and drug-like compounds: the rule-of-five revolution. Drug Discovery Today: Technologies 2004, 1, 337-341, https://doi.org/10.1016/j.ddtec.2004.11.007.

23. Jayaram, B.; Singh, T.; Mukherjee, G.; Mathur, A.; Shekhar, S.; Shekhar, V. Sanjeevini: a freely accessible web-server for target directed lead molecule discovery. BMC Bioinformatics 2012, 13, S7, https://doi.org/10.1186/1471-2105-13-S17-S7. 
24. Sharma, A.; Islam, M.H.; Fatima, N.; Upadhyay, T.K.; Khan, M.K.A.; Dwivedi, U.N.; Sharma, R. Elucidation of marine fungi derived anthraquinones as mycobacterial mycolic acid synthesis inhibitors: an in silico approach. Mol. Biol. Rep. 2019, 46, 1715-1725, https://doi.org/10.1007/s11033-019-04621-0.

25. Neha, S.; Mohammad, F.; Abdulrahman, A.A.; Khan, M.K.A.; Saheem, A.; Salman, A. Design, Sar, and Metabolism Study of Crucifereae Family Compound (Spirobrassinin) and its Analogs for Antiangiogenic $\begin{array}{llllll}\text { Potential Targeting Hsp90. Curr. } & \text { Proteomics } & \mathbf{2 0 2 0}, & 17, & 1-19 \text {, }\end{array}$ http://dx.doi.org/10.2174/1570164617999200731000719.

26. Pierce, B.G.; Wiehe, K.; Hwang, H.; Kim, B.-H.; Vreven, T.; Weng, Z. ZDOCK server: interactive docking prediction of protein-protein complexes and symmetric multimers. Bioinformatics 2014, 30, 1771-1773, https://doi.org/10.1093/bioinformatics/btu097.

27. Santos, K.B.; Guedes, I.A.; Karl, A.L.M.; Dardenne, L.E. Highly Flexible Ligand Docking: Benchmarking of the DockThor Program on the LEADS-PEP Protein-Peptide Data Set. J. Chem. Inf. Model. 2020, 60, 667683, https://doi.org/10.1021/acs.jcim.9b00905.

28. de Magalhães, C.S.; Almeida, D.M.; Barbosa, H.J.C.; Dardenne, L.E. A dynamic niching genetic algorithm strategy for docking highly flexible ligands. Information Sciences 2014, 289, 206-224, https://doi.org/10.1016/j.ins.2014.08.002.

29. Vangone, A.; Bonvin, A.M.J.J. Contacts-based prediction of binding affinity in protein-protein complexes. elife 2015, 4, e07454, https://doi.org/10.7554/eLife.07454.

30. Xue, L.C.; Rodrigues, J.P.; Kastritis, P.L.; Bonvin, A.M.; Vangone, A. PRODIGY: a web server for predicting the binding affinity of protein-protein complexes. Bioinformatics 2016, 32, 3676-3678, https://doi.org/10.1093/bioinformatics/btw514.

31. Pettersen, E.F.; Goddard, T.D.; Huang, C.C.; Couch, G.S.; Greenblatt, D.M.; Meng, E.C.; Ferrin, T.E. UCSF Chimera-A visualization system for exploratory research and analysis. J. Comput. Chem. 2004, 25, 16051612, https://doi.org/10.1002/jcc.20084,

32. Laskowski, R.A.; Swindells, M.B. LigPlot+: Multiple Ligand-Protein Interaction Diagrams for Drug Discovery. J. Chem. Inf. Model. 2011, 51, 2778-2786, https://doi.org/10.1021/ci200227u.

33. Selvaraj, C.; Priya, R.B.; Lee, J.-K.; Singh, S.K. Mechanistic insights of SrtA-LPXTG blockers targeting the transpeptidase mechanism in Streptococcus mutans. RSC Advances 2015, 5, 100498-100510, https://doi.org/10.1039/C5RA12869B.

34. Gunasekaran, D.; Sridhar, J.; Suryanarayanan, V.; Manimaran, N.C.; Singh, S.K. Molecular modeling and structural analysis of $\mathrm{nAChR}$ variants uncovers the mechanism of resistance to snake toxins. J. Biomol. Struct. Dyn. 2017, 35, 1654-1671, https://doi.org/10.1080/07391102.2016.1190791.

35. Shivakumar, D.; Williams, J.; Wu, Y.; Damm, W.; Shelley, J.; Sherman, W. Prediction of Absolute Solvation Free Energies using Molecular Dynamics Free Energy Perturbation and the OPLS Force Field. J. Chem. Theory Comput. 2010, 6, 1509-1519, https://doi.org/10.1021/ct900587b.

36. Olsen, L.; Montefiori, M.; Tran, K.P.; Jørgensen, F.S. SMARTCyp 3.0: enhanced cytochrome P450 site-of$\begin{array}{lllll}\text { metabolism } & \text { prediction } & \text { server. } & \text { Bioinformatics }\end{array}$ https://doi.org/10.1093/bioinformatics/btz037.

37. Hughes, T.B.; Dang, N.L.; Miller, G.P.; Swamidass, S.J. Modeling Reactivity to Biological Macromolecules with a Deep Multitask Network. ACS Central Science 2016, 2, 529-537, https://doi.org/10.1021/acscentsci.6b00162.

38. Dmitriev, A.V.; Filimonov, D.A.; Rudik, A.V.; Pogodin, P.V.; Karasev, D.A.; Lagunin, A.A.; Poroikov, V.V. Drug-drug interaction prediction using PASS. SAR QSAR Environ. Res. 2019, 30, 655-664, https://doi.org/10.1080/1062936X.2019.1653966.

39. Liu, R.; Liu, J.; Tawa, G.; Wallqvist, A. 2D SMARTCyp Reactivity-Based Site of Metabolism Prediction for Major Drug-Metabolizing Cytochrome P450 Enzymes. J. Chem. Inf. Model. 2012, 52, 1698-1712, https://doi.org/10.1021/ci3001524.

40. Tyzack, J.D.; Kirchmair, J. Computational methods and tools to predict cytochrome P450 metabolism for drug discovery. Chem. Biol. Drug Des. 2019, 93, 377-386, https://doi.org/10.1111/cbdd.13445.

41. Heck, Thiago G.; Ludwig, Mirna S.; Frizzo, Matias N.; Rasia-Filho, Alberto A.; Homem de Bittencourt, P.I., Jr. Suppressed anti-inflammatory heat shock response in high-risk COVID-19 patients: lessons from basic research (inclusive bats), light on conceivable therapies. Clin. Sci. 2020, 134, 1991-2017, https://doi.org/10.1042/CS20200596. 
42. Khan, M.K.A.; Akhtar, S.; Arif, J.M. Development of In Silico Protocols to Predict Structural Insights into the Metabolic Activation Pathways of Xenobiotics. Interdisciplinary Sciences: Computational Life Sciences 2018, 10, 329-345, https://doi:10.1007/s12539-017-0237-4.

43. Khan, M.K.A.; Akhtar, S.; Arif, J.M. Structural Insight into the Mechanism of Dibenzo[a,l]pyrene and Benzo $[a]$ pyrene-Mediated Cell Proliferation Using Molecular Docking Simulations. Interdisciplinary Sciences: Computational Life Sciences 2018, 10, 653-673, https://doi.org/10.1007/s12539-017-0226-7.

44. Khan, M.K.A.; Pokharkar, N.B.; Al-Khodairy, F.M.; Al-Marshad, F.M.; Arif, J.M. Structural Perspective on Molecular Interaction of IgG and IgA with Spike and Envelope Proteins of SARS-CoV-2 and Its Implications to Non-Specific Immunity. Biointerface Research in Applied Chemistry 2021, 10923-10939, https://doi.org/10.33263/BRIAC113.1092310939.

45. Khan, F.I.; Lai, D.; Anwer, R.; Azim, I.; Khan, M.K.A. Identifying novel sphingosine kinase 1 inhibitors as therapeutics against breast cancer. J. Enzyme Inhib. Med. Chem. 2020, 35, 172-186, https://doi.org/10.1080/14756366.2019.1692828. 